\title{
Stochastic Dynamics of Feline Immunodeficiency Virus within Cat Populations
}

\author{
Jingli Li ${ }^{a}$, Meijing Shan ${ }^{b}$, Malay Banerjeec ${ }^{c}$ Weiming Wang* a,d \\ ${ }^{a}$ College of Mathematics and Information Science, Wenzhou University, Wenzhou, 325035 P.R. China \\ ${ }^{b}$ Institute of Information Science and Technology, East China University of Political Science and Law, \\ Shanghai, 201620 P.R.China \\ ${ }^{c}$ Department of Mathematics and Statistics, Indian Institute of Technology Kanpur, Kanpur, Uttar \\ Pradesh, India \\ ${ }^{d}$ School of Mathematical Science, Huaiyin Normal University, Huaian, 223300 P.R.China
}

\begin{abstract}
In this paper, we investigate the basic features of a simple susceptible-infected (SI) epidemic model of Feline immunodeficiency virus (FIV) within cat populations in presence of multiplicative noise terms to understand the effects of environmental driving forces on the disease dynamics. The value of this study lies in two aspects. Mathematically, we propose three threshold parameters, $R_{s}^{h}, R_{1}$ and $R_{2}$ to utilize in identifying the stochastic extinction and persistence. In the case of stochastic persistence, we prove that there is a stationary distribution. Based on the statistical data for rural cat populations Barisey-la-Côte in France, we perform some numerical simulations to verify/extend our analytical results. Epidemiologically, we find that: (1) Large environment fluctuations can suppress the outbreak of FIV; (2) The distributions are governed by $R_{s}^{h}$; (3) White noise perturbations of the birth rate for infectious cats (i.e., the vertical transmission) can can induce the susceptible-free dynamics. Keywords: Feline immunodeficiency virus; Extinction; Persistence; Stationary distribution.
\end{abstract}

Email addresses: lijingli20150801@163.com (Jingli Li), shanmeijing@ecupl.edu.cn (Meijing Shan), malayb@ittk.ac.in (Malay Banerjee), weimingwang2003@163.com (Weiming Wang*)

* Author to whom any correspondence should be addressed. 


\section{Introduction}

In 1987, Pedersen and coworkers first described the Feline Immunodeficiency Virus (FIV; formerly feline T-lymphotropic lentivirus) [1], they realized its effectiveness towards the modelling of human immunodeficiency virus (HIV) related disease and its dynamic behavior. FIV was isolated from cats, having immune deficiency syndrome that is similar to human AIDS in various respect [2]. Infection of pathogen-free kittens with FIV resulted in lymphadenopathy. FIV is morphologically similar to HIV-1, and its reverse transcriptase (RT, the retrovirusencoded RNA-dependent DNA polymerase) has a $\mathrm{Mg}^{2+}$ requirement that is similar to that of the HIV-1 RT [3]. And FIV is a member of the lentivirus subfamily and is distinct from characterized retroviruses, which also includes HIV and Simian Immunodeficiency Viruses (SIV) [4]. The expectation that FIV is a leading animal model for HIV studies, indeed, the similarities between these two viruses are so many and, studying FIV will not only help us to understand the pathogenesis of HIV and provide clues for the development of interventive strategies for its control but also will benefit the natural host species [5]. However, FIV is mainly transmitted through bites during aggressive contacts between cats, in contrast to HIV $[6,7]$. Because domestic cat populations live in a variety of ecological setup, they show a wide range of variability in their spatial and social structures which can be compared to other populations of FIV-infected non-domestic fields that are more difficult to study within natural habitats. The wide prevalence of FIV infection in natural populations of cats provides an opportunity to analyse the consequences of population structures on the circulation of the virus related diseases [8].

The mathematical models have been revealed as a powerful tool to understand the dynamic spread of FIV within cat populations $[7,8,9,10,11,12,13]$. In order to describe the spread of FIV through the cat populations, Courchamp et al. [8] proposed a susceptibleinfected (SI) model for the transmission of FIV within domestic cats with logistic growth, the standard incidence rate with no vertical transmission, and found that FIV has a low impact on the population in that the total number of cats at equilibrium point. Nell et al. [9] claimed that vertical transmission of FIV may be a useful modelling approach to eval- 
uate the intervention strategies for HIV transmission from mother to child. Hilker et al. [7] considered the influence of the Allee effect (a phenomenon in biology characterized by a correlation between population size or density and the mean individual fitness of a population or species [14]) on the host population towards the disease spread. Ducrot et al. [13] investigate the global stability of the endemic steady state and the existence of travelling wave solutions connecting the endemic to the disease-free state of an SI type model to FIV with vertical transmission (or call mother-to-child transmission, the transmission of a bacteria, viruses, or in rare cases, parasites transmitted directly from the mother to an embryo, fetus, or baby during pregnancy or childbirth) and a density dependent incidence rate.

In the real world, biological populations exist inevitably under the influence of noisy random environmental condition which affect the environmental parameters and as a result affect the population dynamics significantly. It is well known that in several instances, environmental variations have a significant influence on the development and propagation of an epidemic $[15,16]$. For human disease related epidemics, the nature of epidemic growth and spread is inherently random due to the unpredictability in person-to-person contacts [17] and population is subject to a continuous spectrum of disturbances $[18,19]$. Hence the variability and randomness of the environment is fed through the state of the epidemic [20]. And in epidemic dynamics, stochastic differential equation (SDE) models could be more appropriate way of modeling epidemics under various circumstances $[21,22,23,24,25,26,27,28,29,30$, 31]. However, despite the potential importance of parametric noise, it has received relatively less attention in the research on FIV. And there comes a question: How does environment fluctuations affect the dynamics of FIV within cat populations?

The main focus of this article is to investigate how environment fluctuations introduced to linear growth rates affect disease's dynamics through studying the global dynamics of a general SI-type FIV model for both deterministic and the corresponding stochastic version. The rest of this article is organized as follows: In Section 2, we derive a general SI FIV deterministic model (or without noise) and its corresponding stochastic version (or with noise), and illustrate the main results. In Section 3, we give the proofs of the main results 
in details. In Section 4, we provide some numerical simulations to support our findings. In the last section, we provide a brief discussion and the summary of the main results.

\section{Model derivations and main results}

Throughout this paper, unless otherwise specified, let $\left(\Omega,\left\{\digamma_{t}\right\}_{t \geq 0}, P\right)$ be a complete probability space with a filtration $\left\{\digamma_{t}\right\}_{t \geq 0}$ satisfying the usual conditions (i.e. it is right continuous and $\digamma_{0}$ contains all $P$-null sets).

\subsection{Model derivations}

Following the insightful work of $[7,8,13]$, in this subsection, we establish our model which deals with the spread of FIV through the cat population under assumptions that include the possibility of multiple modes of transmission, that is, horizontal and vertical. First, we give the basic model assumptions as follows:

(i) Assume that the total cat population $N(t)$ is divided into two groups, susceptible (uninfected) cats $S(t)$ and infected cats $I(t)$, i.e., $N(t)=S(t)+I(t)$;

(ii) With regard to the demographic parameters, we assumed that the density dependence acts primarily on mortality, hence the natural birth rate for susceptible cats and infectious

cats can be assumed as constants $b$ and $b_{I}$, respectively. The ratio $0 \leq \rho:=\frac{b_{I}}{b} \leq 1$ describes the reduced reproductive ability of infected cats: $\rho=0$ means that infected cats lose their reproducing ability, that is, in this case, there is no vertical transmission; while $\rho=1$ indicates that they experience no reduction in reproductive fitness. And $0<\rho<1$ means that the FIV is assumed to be transmitted to offspring and implies the vertical transmission. Hence, in this case, newborns of the infected are in the infectious class.

(iii) Assume that the mortality rate of the cat population $N$ is linearly related to $N$ and has the form $m+k N$, where $m$ is the natural death rate and $k=\frac{b-m}{K}$ is a positive real number, here $r:=b-m$ is the intrinsic growth rate of cat populations $N$ in the absence of resource limits, $K$ is the carrying capacity of the population $N$.

(iv) The infected cats suffer an additional FIV-related mortality $\alpha>0$, which shall be referred to as virulence. 
(v) Assume that the rate of horizontal transmission (the transmission of a bacterial, fungal, or viral infection between members of the same species that are not in a parentchild relationship) is proportional to the density of susceptible cats $S$ times the density of infected cats $I$, with proportionality constant $\beta$, a "mass-action" assumption (or called bilinear incidence). Obviously, if $\beta=0$, there is no horizontal transmission.

Such a two-component epidemiological system modelling the spread of the FIV is as follows:

$$
\left\{\begin{array}{l}
\frac{d S}{d t}=b S-(m+k N) S-\beta S I \\
\frac{d I}{d t}=\beta S I-\alpha I+b_{I} I-(m+k N) I,
\end{array}\right.
$$

with initial conditions $S(0)=S_{0}>0, I(0)=I_{0}>0$.

In the absence of FIV, from model (1), we can get:

$$
\frac{d N}{d t}=b N-(m+k N) N=r N\left(1-\frac{N}{\frac{b-m}{k}}\right),
$$

this incorporates the carrying capacity $K=\frac{b-m}{k}$.

To incorporate the effect of environmental fluctuations, here we formulate the stochastic model by introducing two multiplicative noise terms into the growth equations of both the susceptible and infected populations. Following the approach of Mao [21], in this paper, we assume that during the spreading of a disease, the birth rate for susceptible and infectious $b, b_{I}$ will fluctuate randomly around some average value due to continuous fluctuation in the environmental conditions. And we introduce randomness into the deterministic model (1) by perturbing $b$ by $b+\sigma_{1} \zeta(t)$ and $b_{I}$ by $b_{I}+\sigma_{2} \zeta(t)$ to obtain the following stochastic differential equations:

$$
\left\{\begin{array}{l}
\frac{d S}{d t}=\left(b+\sigma_{1} \zeta(t)\right) S-(m+k N) S-\beta S I \\
\frac{d I}{d t}=\beta S I-\alpha I+\left(b_{I}+\sigma_{2} \zeta(t)\right) I-(m+k N) I
\end{array}\right.
$$

where $\zeta(t)$ is a Gaussian white noise and characterized by:

$$
\langle\zeta(t)\rangle=0, \quad\left\langle\zeta(t) \zeta\left(t^{\prime}\right)\right\rangle=\delta\left(t-t^{\prime}\right)
$$


here $\langle\cdot\rangle$ denotes ensemble average and $\delta(\cdot)$ is the Dirac- $\delta$ function. $\sigma_{1}$ and $\sigma_{2}$ denote the intensity of environmental forcing. Now we can rewrite model (2) into the form of stochastic differential equations as follows:

$$
\left\{\begin{array}{l}
d S(t)=[b S-(m+k N) S-\beta S I] d t+\sigma_{1} S d B_{1}(t) \\
d I(t)=\left[\beta S I+b_{I} I-(m+k N) I-\alpha I\right] d t+\sigma_{2} I d B_{2}(t)
\end{array}\right.
$$

where $B_{1}(t)$ and $B_{2}(t)$ are two independent standard one-dimensional Wiener processes defined over the complete probability space $\left(\Omega, \mathcal{F},\left\{\mathcal{F}_{t}\right\}_{t \geq 0}, \mathbb{P}\right)$, the relations between the white noise terms and Wiener process are defined by $d B_{i}(t)=\sigma_{i} \zeta(t) d t(i=1,2)$ where the differentials stand for pathwise derivative.

\subsection{Main results}

One of our main goals in the paper is to derive the FIV dynamics of the deterministic model (1). Following Driessche and Watmough [32], set the basic demographic reproduction number

$$
T_{0}^{d d}=\frac{\beta K}{b+\alpha-b_{I}}, \quad \bar{K}=b_{I}-m-\alpha .
$$

Theorem 2.1. (i) If $T_{0}^{d d}<0$ or $r:=b-m<0$, then for any given initial value $\left(S_{0}, I_{0}\right) \in \mathbb{R}_{+}^{2}$, the whole cat population goes to extinction. That is, $(S(t), I(t))$ tend to $(0,0)$ as $t \rightarrow \infty$.

(ii) If $0<T_{0}^{d d}<1$, then the disease-free steady state DFE $=(K, 0)$ is globally asymptotically stable with respect to initial value $\left(S_{0}, I_{0}\right) \in \mathbb{R}_{+}^{2}$.

(iii) If $T_{0}^{d d}>1$,

(iii-1) if $\bar{K}>0$,

(a) when $\beta \frac{\bar{K}}{k}>b+\alpha-b_{I}$, then the susceptible-free steady state $S F E=\left(0, \frac{\bar{K}}{k}\right)$ is globally asymptotically stable with respect to initial value $\left(S_{0}, I_{0}\right) \in \mathbb{R}_{+}^{2}$;

(b) when $\beta \frac{\bar{K}}{k}<b+\alpha-b_{I}$, then there exists a unique endemic state, $E^{*}=$ $\left(\frac{k\left(b+\alpha-b_{I}\right)-\beta \bar{K}}{\beta^{2}}, \frac{\left(T_{0}^{d d}-1\right)\left(b+\alpha-b_{I}\right) k}{\beta^{2}}\right)$, which is global asymptotic stability for initial data $\left(S_{0}, I_{0}\right) \in$ $\mathbb{R}_{+}^{2}$.

(iii-2) 
if $\bar{K} \leq 0$, then there exists a unique endemic state, $E^{*}=\left(\frac{k\left(b+\alpha-b_{I}\right)-\beta \bar{K}}{\beta^{2}}, \frac{\left(T_{0}^{d d}-1\right)\left(b+\alpha-b_{I}\right) k}{\beta^{2}}\right)$, which is global asymptotic stability for initial data $\left(S_{0}, I_{0}\right) \in \mathbb{R}_{+}^{2}$.

The proof of this theorem is similar to that in [13] and hence is omitted.

The other goal of this paper is to study the FIV dynamics of the stochastic model (2). We define the horizontal transmission reproduction number [32, 29] for the SDE model (3) as:

$$
R_{s}^{h}:=\frac{2 \beta(b-m)+2 k b_{I}-k \sigma_{2}^{2}}{2 k(b+\alpha)} .
$$

Furthermore, for simplicity to show the theoretical results, we define two threshold parameters as:

$$
R_{1}:=b-m-\frac{\sigma_{1}^{2}}{2}
$$

and

$$
R_{2}:=b_{I}-m-\alpha-\frac{\sigma_{2}^{2}}{2}=\bar{K}-\frac{\sigma_{2}^{2}}{2} .
$$

Theorem 2.2. (i) If $R_{1}>0$, when $\beta>k$ and $R_{s}^{h}<1$, or $\beta \leq k$ and $R_{2}<0$ hold, then for any given initial value $\left(S_{0}, I_{0}\right) \in \mathbb{R}_{+}^{2}$, the solution of the SDE model (3) obeys

$$
\begin{gathered}
\limsup _{t \rightarrow \infty} \frac{\log I}{t} \leq(b+\alpha)\left(R_{s}^{h}-1\right)<0 \text { a.s. } \quad \text { or } \quad \limsup _{t \rightarrow \infty} \frac{\log I}{t} \leq \frac{R_{2}}{k}<0 \text { a.s.. } \\
\liminf _{t \rightarrow \infty} \frac{1}{t} \int_{0}^{t} S d s \geq \frac{R_{1}}{k}>0 \text { a.s., }
\end{gathered}
$$

namely, only the infected population $I(t)$ goes to extinction almost surely.

(ii) if $R_{1}<0$,

$$
\text { (ii-1)(a) when } \beta \geq k \text { and } R_{2}>0 \text {, or (b) } \beta<k \text { and } R_{s}^{h}>1
$$

hold, then for any given initial value $\left(S_{0}, I_{0}\right) \in \mathbb{R}_{+}^{2}$, the solution of the SDE model (3) obeys

$$
\limsup _{t \rightarrow \infty} \frac{\log S}{t} \leq R_{1}<0 \quad \text { a.s. }
$$

and

$$
\liminf _{t \rightarrow \infty} \frac{1}{t} \int_{0}^{t} I d s>\frac{R_{2}}{k}>0 \text { a.s. }
$$

namely, only the susceptible $S(t)$ goes to extinction almost surely. 
(ii-2) when $\beta>k$ and $R_{s}^{h}<1$, or $\beta \leq k$ and $R_{2}<0$ hold, then for any given initial value $\left(S_{0}, I_{0}\right) \in \mathbb{R}_{+}^{2}$, the susceptible $S(t)$ and infectious $I(t)$ of the $S D E$ model (3) go to extinction almost surely.

Theorem 2.3. (i) Assume $\beta>k$, if $R_{s}^{h}>1, R_{1}>0$ and $R_{2}>0$ hold, then the solution $(S(t), I(t))$ of the SDE model (3) has the following properties:

$$
\begin{aligned}
& \liminf _{t \rightarrow \infty} \frac{1}{t} \int_{0}^{t} I(s) d s \geq \frac{R_{2}}{k}>0 \quad \text { a.s. } \\
& \limsup _{t \rightarrow \infty} \frac{1}{t} \int_{0}^{t} I(s) d s \leq \frac{(b+\alpha)\left(R_{s}^{h}-1\right)}{k} \quad \text { a.s., }
\end{aligned}
$$

and

$$
0<\limsup _{t \rightarrow \infty} \frac{1}{t} \int_{0}^{t} S(s) d s \leq \frac{R_{1}}{k} \quad \text { a.s.. }
$$

That is, the solutions of model (3) with respect to initial value $\left(S_{0}, I_{0}\right) \in \mathbb{R}_{+}^{2}$ are weakly persist in mean.

(ii) If all assumptions in (i) hold, and assume that $T^{d d}>1$, If $\bar{K} \leq 0$ or $\bar{K}>0$ and $\frac{\beta \bar{K}}{k}<b+\alpha-b_{I}$, and $M_{1}=\frac{1}{2} S^{*} \sigma_{1}^{2}+\frac{1}{2} I^{*} \sigma_{2}^{2}<k\left(S^{*}+I^{*}\right)^{2}$, where $\left(S^{*}, I^{*}\right)$ is the endemic equilibrium of model (1), then there exists a stationary distribution $\mu(\cdot)$ for the SDE model (3).

\section{Proofs of main results}

The following definitions are commonly used and we list them below.

Definition 3.1. [33] The population $x(t)$ is said to go to extinction if $\lim _{t \rightarrow \infty} x(t)=0$.

Definition 3.2. [33] The population $x(t)$ is said to be weakly persistent in mean if

$$
\limsup _{t \rightarrow \infty} \frac{1}{t} \int_{0}^{t} x(s) d s>0 .
$$

Definition 3.3. [33] The population $x(t)$ is said to be strongly persistent in mean if

$$
\liminf _{t \rightarrow \infty} \frac{1}{t} \int_{0}^{t} x(s) d s>0 .
$$


Lemma 3.4. [33] Let $x(t) \in C[\Omega \times[0, \infty),(0, \infty)]$.

(i) If there exist positive constants $\lambda, \gamma$ such that

$$
\log x(t) \leq \lambda t-\gamma \int_{0}^{t} x(s) d s+F(t), \text { a.s. }
$$

for all $t \geq 0$, where $F \in C[\Omega \times[0, \infty), \mathbb{R}]$ and $\lim _{t \rightarrow \infty} \frac{F(t)}{t}=0$, then

$$
\limsup _{t \rightarrow \infty} \frac{1}{t} \int_{0}^{t} x(s) d s \leq \frac{\lambda}{\gamma} \quad \text { a.s.. }
$$

(ii) If there exist positive constants $\lambda, \gamma$ such that

$$
\log x(t) \geq \lambda t-\gamma \int_{0}^{t} x(s) d s+F(t), \text { a.s. }
$$

for all $t \geq 0$, where $F \in C[\Omega \times[0, \infty), \mathbb{R}]$ and $\lim _{t \rightarrow \infty} \frac{F(t)}{t}=0$, then

$$
\liminf _{t \rightarrow \infty} \frac{1}{t} \int_{0}^{t} x(s) d s \geq \frac{\lambda}{\gamma} \quad \text { a.s.. }
$$

\subsection{Existence and uniqueness of the global positive solution}

Theorem 3.5. For any initial condition $\left(S_{0}, I_{0}\right) \in \mathbb{R}_{+}^{2}$, there is a unique solution $(S(t), I(t))$ of the SDE model (3) for all $t \geq 0$ and the solution will remain in $\mathbb{R}_{+}^{2}$ with probability one, namely, $(S(t), I(t)) \in \mathbb{R}_{+}^{2}$ for all $t \geq 0$ almost surely.

Proof. Consider the diffusion process, corresponding to the SDE system (3), as follows

$$
\begin{aligned}
& d u=\left[b-m-k\left(e^{u}+e^{\nu}\right)-\beta e^{\nu}-\frac{1}{2} \sigma_{1}^{2}\right] d t+\sigma_{1} d B_{1}(t), \\
& d \nu=\left[\beta e^{u}+b_{I}-m-k\left(e^{u}+e^{\nu}\right)-\alpha-\frac{1}{2} \sigma_{2}^{2}\right] d t+\sigma_{2} d B_{2}(t),
\end{aligned}
$$

subjected to the initial condition $u(0)=\log S_{0}, \nu(0)=\log I_{0}$. The functions involved with drift part of above stochastic differential system satisfy the linear growth condition and they are locally Lipschitz. Hence there is a unique local solution $(u(t), \nu(t))$, for $t \in\left[0, \tau_{e}\right)$ where $\tau_{e}$ is any finite positive real number. Clearly, the unique positive local solution of model (3) starting from an interior point of the first quadrant $\mathbb{R}_{+}^{2}$ is given by $S(t)=e^{u(t)}, I(t)=e^{\nu(t)}$. 
To show that this solution is global in $\mathbb{R}_{+}^{2}$, we need to show that $\tau_{e}=\infty$ a.s.. We choose a sufficiently large non-negative number $r_{0}$ such that both of $S_{0}$ and $I_{0}$ lie within the interval $\left[\frac{1}{r_{0}}, r_{0}\right]$. For each integer $r \geq r_{0}$, we can define the stopping time

$$
\tau_{r}=\inf \left\{t \in\left[0, \tau_{e}\right): S(t) \notin\left(\frac{1}{r}, r\right) \text { or } I(t) \notin\left(\frac{1}{r}, r\right)\right\},
$$

where $\inf \emptyset=\infty$ (as usual $\emptyset$ denotes the empty set). Clearly, $\tau_{r}$ is increasing as $r \rightarrow \infty$. Set $\tau_{\infty}=\lim _{t \rightarrow \infty} \tau_{r}$, then $\tau_{\infty} \leq \tau_{e}$ a.s..

In the following, we need to show that $\tau_{e}=\infty$ a.s.. If this statement is violated, there exists two constants $T>0$ and $\epsilon \in(0,1)$ such that

$$
P\left\{\tau_{\infty} \leq T\right\}>\epsilon .
$$

Hence we can find an integer $r_{1} \geq r_{0}$ such that

$$
P\left\{\tau_{r} \leq T\right\} \geq \epsilon
$$

for all $r \geq r_{1}$. Define a $C^{2}$-function $V: \mathbb{R}_{+}^{2} \rightarrow \mathbb{R}_{+}$by

$$
V(S, I)=(S+1-\log S)+(I+1-\log I) .
$$

Considering that $(z+1-\log z) \geq 0$ for all $z>0$, the function $V(\cdot)$ is positive definite for all $(S, I) \in \mathbb{R}_{+}^{2}$. Calculating the differential of $V$ along the solution trajectories of the system (3) by using Itô's formula, we get

$$
\begin{gathered}
d V=\left[(b S-(m+k(S+I)) S-\beta S I)\left(1-\frac{1}{S}\right)+\left(\beta S I+b_{I} I-(m+k(S+I)) I-\alpha I\right)\left(1-\frac{1}{I}\right)\right. \\
\left.+\frac{\sigma_{1}^{2}+\sigma_{2}^{2}}{2}\right] d t+\sigma_{1}(S-1) d B_{1}+\sigma_{2}(I-1) d B_{2} .
\end{gathered}
$$

The positivity of $S(t)$ and $I(t)$ implies that

$$
\begin{aligned}
d V & \leq\left[b S-b+2 m+2 k(S+I)+\beta I+b_{I} I-b_{I}+\alpha+\frac{\sigma_{1}^{2}+\sigma_{2}^{2}}{2}\right] d t+\sigma_{1}(S-1) d B_{1}+\sigma_{2}(I-1) d B_{2} . \\
& \leq\left[\left(2 m+\alpha+\frac{\sigma_{1}^{2}+\sigma_{2}^{2}}{2}\right)+(2 k+b) S+\left(\beta+2 k+b_{I}\right) I\right] d t+\sigma_{1}(S-1) d B_{1}+\sigma_{2}(I-1) d B_{2} .
\end{aligned}
$$

We define two positive constants

$$
c_{1}=2 m+\alpha+\frac{\sigma_{1}^{2}+\sigma_{2}^{2}}{2}, \quad c_{2}=\max \left\{4 k+2 b, 4 k+2 \beta+2 b_{I}\right\} .
$$


For $z \geq 0$, using the fact

$$
z \leq 2(z+1-\log z)-(4-2 \log 2)
$$

then we get

$$
(b+2 k) S+\left(\beta+b_{I}+2 k\right) I \leq(2 b+4 k)(S+1-\log S)+\left(4 k+2 \beta+2 b_{I}\right)(I+1-\log I) \leq c_{2} V .
$$

Considering (17) and (18), we can write

$$
d V \leq\left(c_{1}+c_{2} V\right) d t+\sigma_{1}(S-1) d B_{1}+\sigma_{2}(I-1) d B_{2} .
$$

Choose $c_{3}=\max \left\{c_{1}, c_{2}\right\}$, then

$$
d V \leq c_{3}(1+V) d t+\sigma_{1}(S-1) d B_{1}+\sigma_{2}(I-1) d B_{2} .
$$

Therefore, for $t_{1} \leq T$, by doing the integration on both sides of inequality from 0 to $\tau_{r} \wedge t_{1}$, we get

$$
\int_{0}^{\tau_{r} \wedge t_{1}} d V \leq c_{3} \int_{0}^{\tau_{r} \wedge t_{1}}(1+V) d t+\sigma_{1} \int_{0}^{\tau_{r} \wedge t_{1}}(S-1) d B_{1}+\sigma_{2} \int_{0}^{\tau_{r} \wedge t_{1}}(I-1) d B_{2},
$$

where $\tau_{r} \wedge t_{1}=\min \left\{\tau_{r}, t_{1}\right\}$. Using the property of Itô's integral formula [16], we get from the above inequality

$$
V\left(S\left(\tau_{r} \wedge t_{1}\right), I\left(\tau_{r} \wedge t_{1}\right)\right) \leq V\left(S_{0}, I_{0}\right)+c_{3} \int_{0}^{\tau_{r} \wedge t_{1}}(1+V) d t .
$$

Taking expectation of both sides of the above inequality and applying Fubin's theorem $[16,34]$, we can get

$$
\begin{aligned}
E V\left(S\left(\tau_{r} \wedge t_{1}\right), I\left(\tau_{r} \wedge t_{1}\right)\right) & \leq V\left(S_{0}, I_{0}\right)+c_{3} E \int_{0}^{\tau_{r} \wedge t_{1}}(1+V) d t \\
& \leq V\left(S_{0}, I_{0}\right)+c_{3} t_{1}+c_{3} E \int_{0}^{\tau_{r} \wedge t_{1}} V d t \\
& \leq V\left(S_{0}, I_{0}\right)+c_{3} T+c_{3} E \int_{0}^{\tau_{r} \wedge t_{1}} V\left(S\left(\tau_{r} \wedge t_{1}\right), I\left(\tau_{r} \wedge t_{1}\right)\right) d t \\
& =V\left(S_{0}, I_{0}\right)+c_{3} T+c_{3} \int_{0}^{\tau_{r} \wedge t_{1}} E V\left(S\left(\tau_{r} \wedge t_{1}\right), I\left(\tau_{r} \wedge t_{1}\right)\right) d t .
\end{aligned}
$$


Using Gronwall's inequality [23], we get from the inequality above

$$
E V\left(S\left(\tau_{r} \wedge t_{1}\right), I\left(\tau_{r} \wedge t_{1}\right)\right) \leq c_{4}
$$

where $c_{4}=\left(V\left(S_{0}, I_{0}\right)+c_{3} T\right) e^{c_{4} T}$. Set $\Omega_{r}=\left\{\tau_{r} \leq T\right\}$ for $r \geq r_{1}$. So by (16), we have $P\left(\Omega_{r}\right) \geq \epsilon$. Note that for every $\omega \in \Omega_{r}$, there is at least one of $S\left(\tau_{r}, \omega\right), I\left(\tau_{r}, \omega\right)$ which is equal to either $r$ or $\frac{1}{r}$ and hence

$$
V\left(S\left(\tau_{r}\right), I\left(\tau_{r}\right)\right) \geq(r+1-\log r) \wedge\left(\frac{1}{r}+1-\log \frac{1}{r}\right)
$$

Therefore from (15) and (19), it follows that

$$
c_{4} \geq E\left[1_{\Omega_{r}(\omega)} V\left(S\left(\tau_{r}\right), I\left(\tau_{r}\right)\right)\right] \geq \epsilon\left[(r+1-\log r) \wedge\left(\frac{1}{r}+1-\log \frac{1}{r}\right)\right],
$$

where $1_{\Omega_{r}}$ stands for the indicator function of $\Omega_{r}$.

Letting $r \rightarrow \infty$, we get $\infty>c_{4}=\infty$ which leads us to a contradiction. So we must have $\tau_{\infty}=\infty$. This completes the proof.

\subsection{Proof of Theorem 2.2}

In this section, we give the proof of Theorem 2.2, namely, the stochastic extinction. First of all, we give the proof of the extinction of infectious cats $I(t)$, i.e, Theorem 2.2(i).

\subsubsection{Stochastic extinction of the infectious cats $I(t)$}

Proof. For simplicity, here we only prove the case (i-1), and the proof of (i-2) follows similarly.

If $\beta>k$, it is easy to verify that $\limsup _{t \rightarrow \infty} \frac{1}{t} \int_{0}^{t}(S(t)+I(t)) d s \leq \frac{b-m}{k}$ implies for any $\forall \varepsilon>0$, then there is a $T(\omega)>0$ such that $\frac{1}{t} \int_{0}^{t}(S(t)+I(t)) d s \leq \frac{b-m}{k}+\varepsilon$ for $t \geq T(\omega)$. Then

$$
\frac{1}{t} \int_{0}^{t} S(t) d s \leq \frac{b-m}{k}+\varepsilon
$$

By the Itô's formula, we have

$$
d \log I(t)=\left(\beta S+b_{I}-m-k(S+I)-\alpha-\frac{\sigma_{2}^{2}}{2}\right) d t+\sigma_{2} d B_{2}(t)
$$


Integrating it from 0 to $t$ and then dividing both sides by $t$ on the both sides, we have

$$
\begin{aligned}
\frac{\log I}{t} & =\frac{\log I_{0}}{t}+\frac{\beta \int_{0}^{t} S d s}{t}+\left(b_{I}-m-\alpha-\frac{\sigma_{2}^{2}}{2}\right)-\frac{k \int_{0}^{t}(S+I) d s}{t}+\frac{\int_{0}^{t} \sigma_{2} d B_{2}(s)}{t}, \\
& \leq(\beta-k) \frac{\int_{0}^{t} S d s}{t}+\left(b_{I}-m-\alpha-\frac{\sigma_{2}^{2}}{2}\right)-\frac{k \int_{0}^{t} I d s}{t}+\phi_{1}(t) \\
& \leq(\beta-k)\left(\frac{b-m}{k}+\varepsilon\right)+b_{I}-m-\alpha-\frac{\sigma_{2}^{2}}{2}+\phi_{1}(t),
\end{aligned}
$$

where $\phi_{1}(t)=\frac{\log I_{0}}{t}+\frac{\int_{0}^{t} \sigma_{2} d B_{2}}{t}$, and $\lim _{t \rightarrow \infty} \phi_{1}(t)=0$ a.s. according to the strong law of large numbers for martingales [23]. According to Lemma 3.4 (i) and $\varepsilon$ arbitrary, we can get

$$
\limsup _{t \rightarrow \infty} \frac{\log I}{t} \leq \frac{\beta(b-m)}{k}+b_{I}-b-\alpha-\frac{\sigma_{2}^{2}}{2}=(b+\alpha)\left(R_{s}^{h}-1\right)<0 \quad \text { a.s.. }
$$

so, there exists some constants $\lambda>0$ such that

$$
\limsup _{t \rightarrow \infty} \frac{\log I}{t} \leq \frac{\beta(b-m)}{k}+b_{I}-b-\alpha-\frac{\sigma_{2}^{2}}{2}=(b+\alpha)\left(R_{s}^{h}-1\right) \leq-\lambda<0 \quad \text { a.s.. }
$$

Hence, $\limsup _{t \rightarrow \infty} \frac{\log I}{t} \leq-\lambda$ implies that for any $\varepsilon_{1}>0$, there is a $T\left(\omega_{1}\right)>0$ such that $\frac{\log I}{t} \leq-\lambda+\varepsilon_{1}$ for all $t \geq T\left(\omega_{1}\right)$. Hence we have

$$
I(t) \leq e^{-\lambda t+\varepsilon_{1}}
$$

By the Itô's formula, we have

$$
d \log S=\left(b-m-k(S+I)-\beta I-\frac{\sigma_{1}^{2}}{2}\right) d t+\sigma_{1} d B_{1} .
$$

Integrating it from 0 to $t$ and dividing by $t$ on the both sides, then from (24), we have

$$
\begin{aligned}
\frac{\log S}{t} & =\frac{\log S_{0}}{t}+\left(b-m-\frac{\sigma_{1}^{2}}{2}\right)-\frac{k}{t} \int_{0}^{t}(S+I) d s-\frac{\beta}{t} \int_{0}^{t} I d s+\frac{\int_{0}^{t} \sigma_{1} d B_{1}(s)}{t} \\
& \geq \frac{\log S_{0}}{t}+\left(b-m-\frac{\sigma_{1}^{2}}{2}\right)-(k+\beta) \frac{\int_{0}^{t} e^{-\lambda t+\varepsilon_{1}} d s}{t}-\frac{k}{t} \int_{0}^{t} S d s+\frac{\int_{0}^{t} \sigma_{1} d B_{1}(s)}{t}, \\
& \geq b-m-\frac{\sigma_{1}^{2}}{2}-\frac{k}{t} \int_{0}^{t} S d s+\phi_{2}(t)
\end{aligned}
$$

where

$$
\phi_{2}(t)=\frac{\log S_{0}}{t}-(k+\beta) \frac{\int_{0}^{t} e^{-\lambda t+\varepsilon_{1}} d s}{t}+\frac{\int_{0}^{t} \sigma_{1} d B_{1}(s)}{t}
$$


As we can choose $\varepsilon_{1}$ small enough, according to the strong law of large numbers for martingales [23], we get $\lim _{t \rightarrow \infty} \phi_{2}(t)=0$ a.s..

From lemma 3.4 (ii), we have

$$
\liminf _{t \rightarrow \infty} \frac{1}{t} \int_{0}^{t} S d s \geq \frac{R_{1}}{k}>0
$$

This completes the proof of (i).

Remark 3.6. Theorem 2.2 talks about the case that only infected compartment $I(t)$ goes to extinction almost surely. Therefore a natural question arises: in this situation, what will be the limiting magnitude of the susceptible compartment $S(t)$ ?

In fact, when $P\left\{\lim _{t \rightarrow \infty} I(t)=0\right\}=1$, then from the SDE (3), we get the following limiting equation

$$
d S=\left[(b-m) S-k S^{2}\right] d t+\sigma_{1} S d B_{1} .
$$

The distribution of $S(t)$ converges to a stationary distribution which has a density function [15]

$$
P(S)=\frac{\left(\frac{2 k}{\sigma_{1}^{2}}\right)^{2(b-m) / \sigma_{1}^{2}-1}}{\Gamma\left(\frac{2(b-m)}{\sigma_{1}^{2}}-1\right)} S^{\frac{2(b-m)}{\sigma_{1}^{2}}-2} e^{\frac{-2 k S}{\sigma_{1}^{2}}}
$$

for $\sigma_{1}^{2}<2(b-m)$, where $\Gamma(\mu)=\int_{0}^{\infty} t^{\mu-1} e^{-t} d t$, hence the mean of density $P(S)$ is

$$
E(S)=\frac{b-m}{k}-\frac{\sigma_{1}^{2}}{2 k}
$$

It is easy to verify that,

$$
\lim _{\sigma_{1} \rightarrow 0} E(S)=\lim _{\sigma_{1} \rightarrow 0}\left(\frac{b-m}{k}-\frac{\sigma_{1}^{2}}{2 k}\right)=\frac{b-m}{k},
$$

which means that the mean of $S(t)$ will tend to $K=\frac{b-m}{k}$. That is, the dynamics of the disease-free situation for the stochastic model is same as the result provided in Theorem 2.1(iii)(a) for the deterministic model (1). 


\subsubsection{Stochastic extinction of the susceptible cats $S(t)$}

We now focus on the proof of Theorem 2.2(ii-1).

Proof. Here also we will prove the case (ii-1)(a) only, the proof of (b) can be carried out in similar fashion. If $\beta \geq k$, from (22), we get

$$
\begin{aligned}
\frac{\log I}{t} & =\frac{\log I_{0}}{t}+\frac{\beta}{t} \int_{0}^{t} S d s+\left(b_{I}-m-\alpha-\frac{\sigma_{2}^{2}}{2}\right)-\frac{k}{t} \int_{0}^{t}(S+I) d s+\frac{\int_{0}^{t} \sigma_{2} d B_{2}(s)}{t} \\
& \geq b_{I}-m-\alpha-\frac{\sigma_{2}^{2}}{2}-\frac{k}{t} \int_{0}^{t} I d s+\phi_{1}(t),
\end{aligned}
$$

where $\phi_{1}(t)=\frac{\log I_{0}}{t}+\frac{\int_{0}^{t} \sigma_{2} d B_{2}}{t}$, and $\lim _{t \rightarrow \infty} \phi_{1}(t)=0$ a.s.. From Lemma 3.4 (ii), we get

$$
\liminf _{t \rightarrow \infty} \frac{1}{t} \int_{0}^{t} I d s \geq \frac{b_{I}-m-\alpha-\frac{\sigma_{2}^{2}}{2}}{k}=\frac{R_{2}}{k}>0 \quad \text { a.s.. }
$$

On the other hand, considering (25) and (27) together, we get

$$
\begin{aligned}
\frac{\log S}{t} & =\frac{\log S_{0}}{t}+\left(b-m-\frac{\sigma_{1}^{2}}{2}\right)-\frac{k}{t} \int_{0}^{t}(S+I) d s-\frac{\beta}{t} \int_{0}^{t} I d s+\frac{\int_{0}^{t} \sigma_{1} d B_{1}(s)}{t}, \\
& \leq b-m-\frac{\sigma_{1}^{2}}{2}+\phi_{3}(t),
\end{aligned}
$$

where $\phi_{3}(t)=\frac{\log S_{0}}{t}+\frac{\int_{0}^{t} \sigma_{1} d B_{1}(s)}{t}$, and $\lim _{t \rightarrow \infty} \phi_{3}(t)=0$ a.s., we have

$$
\limsup _{t \rightarrow \infty} \frac{\log S}{t} \leq b-m-\frac{\sigma_{1}^{2}}{2}=R_{1}<0 \quad \text { a.s.. }
$$

The proof of the first part is completed.

Remark 3.7. Theorem 2.2(ii-1) shows the susceptible-free dynamics of the SDE model (3). If $\lim _{t \rightarrow \infty} S(t)=0$ a.s., we can get the limiting equation

$$
d I=\left[\left(b_{I}-m-\alpha\right) I-k I^{2}\right] d t+\sigma_{2} I d B_{2} .
$$

And the distribution of the process $I(t)$ converges to a stationary distribution which has a density [15]

$$
P(I)=\frac{\left(\frac{2 k}{\sigma_{2}^{2}}\right)^{2\left(b_{I}-m-\alpha\right) / \sigma_{2}^{2}-1}}{\Gamma\left(\frac{2\left(b_{I}-m-\alpha\right)}{\sigma_{2}^{2}}-1\right)} I^{2\left(b_{I}-m-\alpha\right) / \sigma_{2}^{2}-2} e^{-2 k \sigma_{2}^{2} I},
$$


for $\sigma_{2}^{2}<2\left(b_{I}-m-\alpha\right)$, where $\Gamma(\mu)=\int_{0}^{\infty} t^{\mu-1} e^{-t} d t$, hence the mean of density $P(I)$ is

$$
E(I)=\frac{b_{I}-m-\alpha}{k}-\frac{\sigma_{2}^{2}}{2 k}
$$

It is easy to verify the limiting dynamics as follows,

$$
\lim _{\sigma_{2} \rightarrow 0} E(I)=\lim _{\sigma_{2} \rightarrow 0}\left(\frac{b_{I}-m-\alpha}{k}-\frac{\sigma_{2}^{2}}{2 k}\right)=\frac{b_{I}-m-\alpha}{k} .
$$

Here also the susceptible-free dynamics within the stochastic setup is analogous to the deterministic dynamics, the relevant result is provided at Theorem 2.1(iii)(b).

Remark 3.8. The proof of Theorem 2.2(ii-2) (i.e., the property of stochastic extinction of the cat population $N(t)$ ) is similar to that in Theorem 2.2(i) or (ii-1) and hence omitted.

\subsection{Proof of Theorem 2.3}

Now, we give the proof of Thereom 2.3, namely, the persistence of the infectious $I(t)$ and the susceptible $S(t)$ (or called endemic) and the existence of the stationary distribution. Firstly, we prove Theorem 2.3(i).

\subsubsection{Persistence of the infectious cats and the susceptible cats}

Proof. Assume $\beta>k$, Set $V=\log I$, by Itô's formula for (3), we have

$$
\begin{aligned}
\frac{\log I}{t} & =\frac{\log I_{0}}{t}+\frac{\beta}{t} \int_{0}^{t} S d s+\left(b_{I}-m-\alpha-\frac{\sigma_{2}^{2}}{2}\right)-\frac{k}{t} \int_{0}^{t}(S+I) d s+\frac{\int_{0}^{t} \sigma_{2} d B_{2}(s)}{t} \\
& =\frac{\log I_{0}}{t}+\frac{(\beta-k)}{t} \int_{0}^{t} S d s+\left(b_{I}-m-\alpha-\frac{\sigma_{2}^{2}}{2}\right)-\frac{k}{t} \int_{0}^{t} I d s+\frac{\int_{0}^{t} \sigma_{2} d B_{2}(s)}{t} \\
& \geq\left(b_{I}-m-\alpha-\frac{\sigma_{2}^{2}}{2}\right)-\frac{k}{t} \int_{0}^{t} I d s+\phi_{1}(t),
\end{aligned}
$$

where $\phi_{1}(t)=\frac{\log I_{0}}{t}+\frac{\int_{0}^{t} \sigma_{2} d B_{2}}{t}$ and $\lim _{t \rightarrow \infty} \phi_{1}(t)=0$ a.s.. Then from Lemma 3.4 (ii), we get

$$
\liminf _{t \rightarrow \infty} \frac{1}{t} \int_{0}^{t} I(s) d s \geq \frac{b_{I}-m-\alpha-\sigma_{2}^{2} / 2}{k}=\frac{R_{2}}{k}>0 \text { a.s.. }
$$


In addition,

$$
\begin{aligned}
\frac{\log I}{t} & =\frac{\log I_{0}}{t}+\frac{\beta}{t} \int_{0}^{t} S d s+\left(b_{I}-m-\alpha-\frac{\sigma_{2}^{2}}{2}\right)-\frac{k}{t} \int_{0}^{t}(S+I) d s+\frac{\int_{0}^{t} \sigma_{2} d B_{2}(s)}{t} \\
& \leq \frac{\log I_{0}}{t}+\frac{(\beta-k)}{t} \int_{0}^{t} S d s+b_{I}-m-\alpha-\frac{\sigma_{2}^{2}}{2}-\frac{k}{t} \int_{0}^{t} I d s+\frac{\int_{0}^{t} \sigma_{2} d B_{2}(s)}{t} \\
& \leq \frac{\log I_{0}}{t}+(\beta-k)\left(\frac{b-m}{k}+\varepsilon\right)+b_{I}-m-\alpha-\frac{\sigma_{2}^{2}}{2}-\frac{k}{t} \int_{0}^{t} I d s+\frac{\int_{0}^{t} \sigma_{2} d B_{2}(s)}{t} \\
& \leq \frac{\beta(b-m)}{k}+b_{I}-b-\alpha-\frac{\sigma_{2}^{2}}{2}-\frac{k}{t} \int_{0}^{t} I d s+\phi_{4}(t),
\end{aligned}
$$

where $\phi_{4}(t)=\frac{\log I_{0}}{t}+(\beta-k) \varepsilon+\frac{\int_{0}^{t} \sigma_{2} d B_{2}(s)}{t}$, and when $\varepsilon$ is small enough, according to the strong law of large numbers for martingales [23], we have

$$
\lim _{t \rightarrow \infty} \phi_{4}(t)=0 \quad \text { a.s.. }
$$

From Lemma 3.4 (i), we have

$$
\limsup _{t \rightarrow \infty} \frac{1}{t} \int_{0}^{t} I(s) d s \leq \frac{\frac{\beta(b-m)}{k}+b_{I}-b-\alpha-\sigma_{2}^{2} / 2}{k}=\frac{(b+\alpha)\left(R_{s}^{h}-1\right)}{k} \quad \text { a.s.. }
$$

And according to (25), we can get

$$
\begin{aligned}
\frac{\log S}{t} & =\frac{\log S_{0}}{t}+\left(b-m-\frac{\sigma_{1}^{2}}{2}\right)-\frac{k}{t} \int_{0}^{t}(S+I) d s-\frac{\beta}{t} \int_{0}^{t} I d s+\frac{\int_{0}^{t} \sigma_{1} d B_{1}(s)}{t} \\
& \leq \frac{\log S_{0}}{t}+b-m-\frac{\sigma_{1}^{2}}{2}-\frac{k}{t} \int_{0}^{t} S d s+\frac{\int_{0}^{t} \sigma_{1} d B_{1}(s)}{t} \\
& \leq b-m-\frac{\sigma_{1}^{2}}{2}-\frac{k}{t} \int_{0}^{t} S d s+\phi_{2}(t),
\end{aligned}
$$

where $\phi_{2}(t)=\frac{\log S_{0}}{t}+\frac{\int_{0}^{t} \sigma_{1} d B_{1}(s)}{t}$ and $\lim _{t \rightarrow \infty} \phi_{2}(t)=0$ a.s.. Then according to lemma 3.4 (i), we can get

$$
\limsup _{t \rightarrow \infty} \frac{1}{t} \int_{0}^{t} S(s) d s \leq \frac{b-m-\frac{\sigma_{1}^{2}}{2}}{k}=\frac{R_{1}}{k} \text { a.s.. }
$$

Besides, taking the limit superior in (25), we can get

$$
\frac{\log S / S_{0}}{t}=\left(b-m-\frac{\sigma_{1}^{2}}{2}\right)-\frac{k}{t} \int_{0}^{t} S d s-\frac{(k+\beta)}{t} \int_{0}^{t} I d s+\frac{\int_{0}^{t} \sigma_{1} d B_{1}(s)}{t} .
$$


In fact, $\limsup _{t \rightarrow \infty} \frac{\log S / S_{0}}{t} \leq 0, \lim _{t \rightarrow \infty} \frac{\int_{0}^{t} \sigma_{1} d B_{1}(s)}{t}=0$ a.s., hence

$$
\begin{aligned}
& \frac{k}{t} \int_{0}^{t} S d s+\frac{(k+\beta)}{t} \int_{0}^{t} I d s \\
& \geq \limsup _{t \rightarrow \infty} \frac{\log S}{t}+\frac{k}{t} \int_{0}^{t} S d s+\frac{(k+\beta)}{t} \int_{0}^{t} I d s-\liminf _{t \rightarrow \infty} \frac{\int_{0}^{t} \sigma_{1} d B_{1}(s)}{t} \\
& \geq b-m-\frac{\sigma_{1}^{2}}{2}=R_{1}>0
\end{aligned}
$$

Thus

$$
\limsup _{t \rightarrow \infty} \frac{1}{t} \int_{0}^{t} S d s>0 \quad \text { a.s.. }
$$

And for $\forall \omega_{1} \in\left\{\limsup _{t \rightarrow \infty} \frac{\int_{0}^{t} S\left(s, \omega_{1}\right) d s}{t}=0\right\}$, it follows from (35) that

$$
\limsup _{t \rightarrow \infty} \frac{\int_{0}^{t} I\left(s, \omega_{1}\right) d s}{t}>0
$$

On the other hand, taking the limit superior in (22) and then using $\limsup _{t \rightarrow \infty} \frac{\int_{0}^{t} S(s) d s}{t}=0$, we can get

$$
\limsup _{t \rightarrow \infty} \frac{\log I\left(t, \omega_{1}\right)}{t} \leq b_{I}-m-\alpha-\frac{\sigma_{2}^{2}}{2}-k \liminf _{t \rightarrow \infty} \frac{1}{t} \int_{0}^{t} I(s) d s \leq 0 .
$$

Consider $I\left(s, \omega_{1}\right) \geq 0$, then

$$
\limsup _{t \rightarrow \infty} \frac{\int_{0}^{t} I\left(s, \omega_{1}\right) d s}{t}=0 .
$$

This is a contradiction. Therefore, we must have $\limsup _{t \rightarrow \infty} \frac{1}{t} \int_{0}^{t} S(s) d s>0$ a.s..

The proof is completed.

Theorem 3.9. Assume $\beta \leq k$, If $R_{s}^{h}>1, R_{1}>0$ and $R_{2}>0$ hold, then the solutions of model (3) with respect to initial value $\left(S_{0}, I_{0}\right) \in \mathbb{R}_{+}^{2}$ are weakly persistent in mean.

The proof of Theorem 3.9 is similar to that in Theorem 2.3(i) and hence is omitted. 


\subsubsection{Existence of stationary distribution}

For the sake of understanding of the stochastic endemic dynamics of the SDE model (3), we now focus on the existence of stationary distribution. We firstly state a useful lemma from Ref. [35] which will be useful to obtain the main result.

Let $X(t)$ be homogeneous Markov process defined over the $l$-dimensional Euclidean space, denoted by $E_{l}$ and is described by the following system of stochastic differential equations:

$$
d X(t)=A(X) d t+\sum_{r=1}^{l} f_{r}(X) d B_{r}(t) .
$$

The diffusion matrix is defined by [35]:

$$
A(x)=\left(a_{i j}(x)\right), \quad a_{i j}(x)=\sum_{r=1}^{l} f_{r}^{i} f_{r}^{j}(x) .
$$

We assume there exists a bounded domain $U \subset E_{l}$ with regular boundary $\Gamma$ and satisfies the following properties:

(H1) In the domain $U$ and some neighborhood thereof, the smallest eigenvalue of the diffusion matrix $A(x)$ is bounded away from zero.

(H2) If $x \in E_{l} \backslash U$, the mean time $\tau$ at which a path emerging from $x$ reaches the set $U$ is finite, and $\sup _{x \in Q} E_{x} \tau<\infty$ for every compact subset $Q \subset E_{l}$.

Lemma 3.10. [35] If the assumptions (H1) and (H2) hold, then the Markov process X(t) has a stationary distribution $\mu(\cdot)$. Let $g(\cdot)$ be a integrable function with respect to the measure $\mu$, then

$$
P_{x}\left\{\lim _{T \rightarrow \infty} \frac{1}{T} \int_{0}^{T} g(X(t)) d t=\int_{E_{l}} g(x) \mu(d x)\right\}=1
$$

for all $x \in E_{l}$.

Remark 3.11. For model (3), the diffusion matrix is

$$
A=\operatorname{diag}\left(\sigma_{1}^{2} S^{2}, \sigma_{2}^{2} I^{2}\right)
$$

\section{Proof of Theroem 2.3(ii)}


Proof. Since $T^{d d}>1, \bar{K} \leq 0$ or $\bar{K}>0$ and $\frac{\beta \bar{K}}{k}<b+\alpha-b_{I}$, then there exists a positive equilibrium point of model (1). Hence

$$
b-m=k\left(S^{*}+I^{*}\right)+\beta I^{*}, \quad b_{I}-m-\alpha=k\left(S^{*}+I^{*}\right)-\beta S^{*} .
$$

Define a positive definite function $V: \mathbb{R}_{+}^{2} \rightarrow \mathbb{R}_{+}$as follows:

$$
V(S, I)=\left(S-S^{*}-S^{*} \log \left(S / S^{*}\right)\right)+\left(I-I^{*}-I^{*} \log \left(I / I^{*}\right)\right) \equiv V_{1}+V_{2} .
$$

Applying Itô's formula and using (38), we can get

$$
\begin{aligned}
d V_{1} & =\left[\left(S-S^{*}\right)(b-m-k(S+I)-\beta I)+\frac{1}{2} S^{*} \sigma_{1}^{2}\right] d t+\sigma_{1}\left(S-S^{*}\right) d B_{1} \\
& =\left[\left(S-S^{*}\right)\left(k\left(S^{*}+I^{*}\right)+\beta I^{*}-k(S+I)-\beta I\right)+\frac{1}{2} S^{*} \sigma_{1}^{2}\right] d t+\sigma_{1}\left(S-S^{*}\right) d B_{1} \\
& =\left[\left(S-S^{*}\right)\left(k\left(S^{*}-S\right)-(k+\beta)\left(I-I^{*}\right)\right)+\frac{1}{2} S^{*} \sigma_{1}^{2}\right] d t+\sigma_{1}\left(S-S^{*}\right) d B_{1} \\
& =\left[-k\left(S-S^{*}\right)^{2}-(k+\beta)\left(S-S^{*}\right)\left(I-I^{*}\right)+\frac{1}{2} S^{*} \sigma_{1}^{2}\right] d t+\sigma_{1}\left(S-S^{*}\right) d B_{1},
\end{aligned}
$$

and

$$
\begin{aligned}
d V_{2} & =\left[\left(I-I^{*}\right)\left(\beta S+b_{I}-m-\alpha-k(S+I)\right)+\frac{1}{2} I^{*} \sigma_{2}^{2}\right] d t+\sigma_{2}\left(I-I^{*}\right) d B_{2} \\
& =\left[\left(I-I^{*}\right)\left(\beta S-\beta S^{*}+k\left(S^{*}+I^{*}\right)-k(S+I)\right)+\frac{1}{2} I^{*} \sigma_{2}^{2}\right] d t+\sigma_{2}\left(I-I^{*}\right) d B_{2} \\
& =\left[-k\left(I-I^{*}\right)^{2}+(\beta-k)\left(S-S^{*}\right)\left(I-I^{*}\right)+\frac{1}{2} I^{*} \sigma_{2}^{2}\right] d t+\sigma_{2}\left(I-I^{*}\right) d B_{2} .
\end{aligned}
$$

Therefore

$$
d V=d V_{1}+d V_{2}=L V d t+\sigma_{1}\left(S-S^{*}\right) d B_{1}+\sigma_{2}\left(I-I^{*}\right) d B_{2},
$$

where

$$
\begin{aligned}
L V= & -k\left(S-S^{*}\right)^{2}-(k+\beta)\left(S-S^{*}\right)\left(I-I^{*}\right)-k\left(I-I^{*}\right)^{2}+(\beta-k)\left(S-S^{*}\right)\left(I-I^{*}\right) \\
& +\frac{1}{2} S^{*} \sigma_{1}^{2}+\frac{1}{2} I^{*} \sigma_{2}^{2} .
\end{aligned}
$$


Set $M_{1}=\frac{1}{2} S^{*} \sigma_{1}^{2}+\frac{1}{2} I^{*} \sigma_{2}^{2}$, then

$$
\begin{aligned}
L V & =-k\left(S-S^{*}\right)^{2}-2 k\left(S-S^{*}\right)\left(I-I^{*}\right)-k\left(I-I^{*}\right)^{2}+M_{1} \\
& \leq-k\left(S-S^{*}\right)^{2}+2 k\left|S-S^{*}\right|\left|I-I^{*}\right|-k\left(I-I^{*}\right)^{2}+M_{1} \\
& =-k\left(\left|S-S^{*}\right|+\left|I-I^{*}\right|\right)^{2}+M_{1} .
\end{aligned}
$$

Now if $M_{1}$ satisfies the following condition,

$$
M_{1}<k\left(S^{*}+I^{*}\right)^{2}
$$

then the ellipse

$$
k\left(\left|S-S^{*}\right|+\left|I-I^{*}\right|\right)^{2}=M_{1}
$$

lies entirely in $\mathbb{R}_{+}^{2}$. We can take $U$ to be a neighborhood of the ellipse with $\bar{U} \subseteq E_{2}=\mathbb{R}_{+}^{2}$, where $\bar{U}$ is the compact closure of $U$. so for $x \in U \backslash E_{2}, L V<0$, which implies conditions (ii) in Lemma (3.10).

Besides, there is

$$
M=\min \left\{\sigma_{1}^{2} S^{2}+\sigma_{2}^{2} I^{2},(S, I) \in \bar{U}\right\}>0
$$

such that

$$
\sum_{i, j=1}^{2} a_{i j} \xi_{i} \xi_{j}=\sigma_{1}^{2} S^{2} \xi_{i}^{2}+\sigma_{2}^{2} I^{2} \xi_{j}^{2} \geq M|\xi|^{2},
$$

for all $(S, I) \in \bar{U}, \xi \in \mathbb{R}_{+}^{2}$, which shows that conditions (i) of lemma (3.10) is also satisfied.

Therefore we can conclude that the SDE model (3) has a stationary distribution $\mu(\cdot)$.

\section{Numerical simulations and dynamics comparisons}

In this section, we provide some numerical simulation results to compare disease dynamics resulting from the deterministic model (1) (in the case of $\sigma_{1}=\sigma_{2}=0$ in model (3)) with its stochastic counterpart (3) for same set of parameter values. Here we use the Milstein's method [36] to simulate the stochastic model (3). The numerical scheme for the Milstein's 
method applied to the stochastic model (3) under considerations is given by,

$$
\left\{\begin{array}{l}
S_{j+1}=S_{j}+\left[b S_{j}-\left(m+k\left(S_{j}+I_{j}\right)\right) S_{k}-\beta S_{j} I_{j}\right] \triangle t+\sigma_{1} \epsilon_{1 j} \sqrt{\triangle t}+\frac{\sigma_{1}^{2}}{2} \triangle t\left(\epsilon_{1 j}^{2}-1\right), \\
I_{j+1}=I_{j}+\left[\beta S_{j} I_{j}+b_{I} I-\left(m+k\left(S_{j}+I_{j}\right)\right) I_{j}-\alpha I\right] \triangle t+\sigma_{2} \epsilon_{2 j} \sqrt{\triangle t}+\frac{\sigma_{2}^{2}}{2} \triangle t\left(\epsilon_{2 j}^{2}-1\right) .
\end{array}\right.
$$

where $\epsilon_{1 j}$ and $\epsilon_{2 j}$ are two independent Gaussian random variables $N(0,1)$ for $j=1,2, \ldots, n$.

The parameter values are chosen as follows (See, Table 1 for details). In rural areas, preliminary observations led us to a value of 2.4 for fecundity $b$ and the mortality rate $m$, per cat, at 0.6 year. The virulence $\alpha=0.2$ year $^{-1}$ is kept constant. The carrying capacity of the habitat is $K=46[8,37]$. Consider the vertical transmission, the transmission coefficient has been estimated to be approximately 3.0/N(0) year $^{-1}$. And the initial values $N(0)$ can be adopted the data for rural cat populations (Barisey-la-Côte) in France (See, Table 1 in [8]):

$$
(S(0), I(0))=(30,3) \in R_{+}^{2} .
$$

Hence, $N(0)=S(0)+I(0)=33$ and $\beta=\frac{3}{33} \approx 0.09091$.

Under these parameters above, we can easily calculate that if $b_{I} \geq 1.34$, model (1) has no positive equilibrium. Hence, we can conclude that $0<b_{I}<1.34$. As an example, we adopt $b_{I}=1.0$ in the numerical simulations.

Table 1: Parameter values in numerical simulations for model (3)

\begin{tabular}{lll}
\hline Parameters & Value range & Reference \\
\hline$b:$ The birth rate for susceptible cats & 2.4 year $^{-1}$ & {$[8,7,37]$} \\
$b_{I}:$ The birth rate for infectious cats & 1.0 year $^{-1}$ & Estimated \\
$m:$ The natural death rate & 0.6 year $^{-1}$ & {$[8,7,37]$} \\
$K:$ The carrying capacity of the habitat & $46 \mathrm{~km}^{-2}$ & {$[8]$} \\
$\alpha:$ The FIV-related mortality & 0.2 year $^{-1}$ & {$[8,7]$} \\
$\beta:$ The transmission coefficient & $\frac{3.0}{N(0)}$ year $^{-1}$ & {$[8,7]$} \\
\hline
\end{tabular}

For this choice of the parameters, we can calculate for the deterministic model (1), $T_{0}^{d d}=2.6137, \bar{K}=b_{I}-m-\alpha=0.2>0$ and $\beta \frac{\bar{K}}{k}=0.4647<b+\alpha-b_{I}=1.6$. Hence, according 
to Theorem 2.1(iii), for model (1), all the trajectories eventually approach towards the endemic equilibrium point $E^{*}=(5.3756,12.2244)$ which is globally asymptotically stable. In addition, the susceptible-free equilibrium $\mathrm{SFE}=(0,5.1111)$ and the disease-free equilibrium $D F E=(46,0)$ are saddle points, $(0,0)$ is a nodal point (See, Fig. 1).

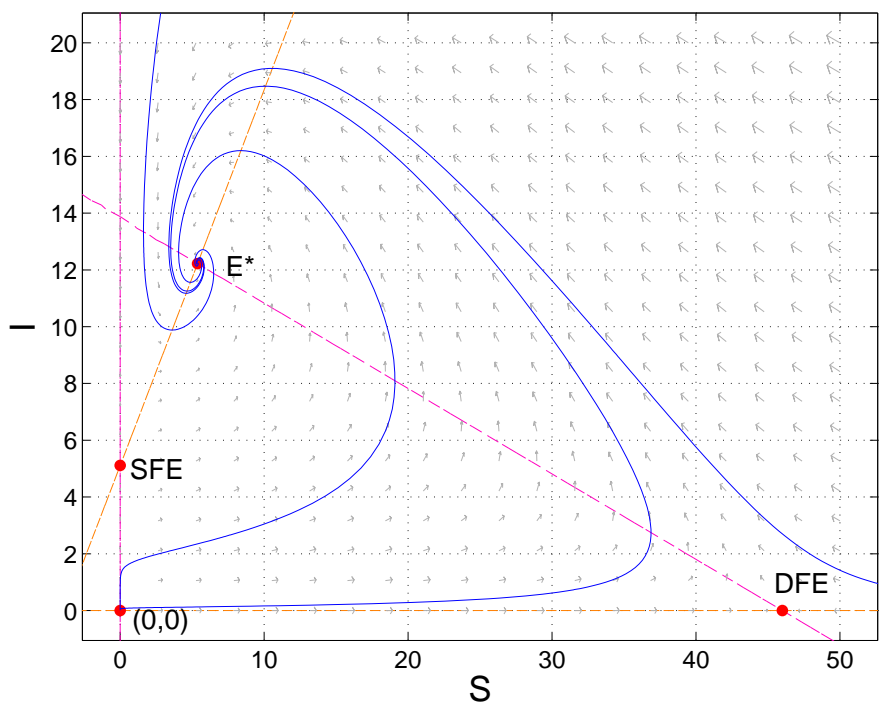

Figure 1: The phase portrait of model (1). The parameters are taken as $\beta=0.09091, b=2.4, k=$ $0.03913, m=0.6, \alpha=0.2, b_{I}=1$. The endemic equilibrium point $E^{*}=(5.3756,12.2244)$ is globally asymptotically stable. In this case, the susceptible-free equilibrium $\operatorname{SFE}=(0,5.1111)$ and the disease-free equilibrium $D F E=(46,0)$ are all saddle points, and the extinction state $(0,0)$ is a nodal point.

Next we focus on the role of noise strength on the resulting dynamics for the SDE model (3). For simplicity, we fix $\sigma_{1}=0.1$, and consider three different values of $\sigma_{2}$ : 0.1 , 0.5 and 2.3. The corresponding values of $R_{s}^{h}$ are 1.9911, 1.9449 and 0.9757 , respectively. By Theorem 2.2 we can know that the FIV is persistent in the first two cases while the infectious $I(t)$ is extinction in the last case. The numerical simulations shown in Figures 2 clearly support these results.

Figure 2 (LEFT) shows the evolution of a single path of $I(t)$ for system (3) and its corresponding deterministic model with three different values of $\sigma_{2}: 0.1,0.5$ and 2.3. One can see that, in the case of FIV persistence, i.e., $\sigma_{2}=0.1$ (See Fig. 2(a)) and 0.5 (See Fig. 2(b)), 

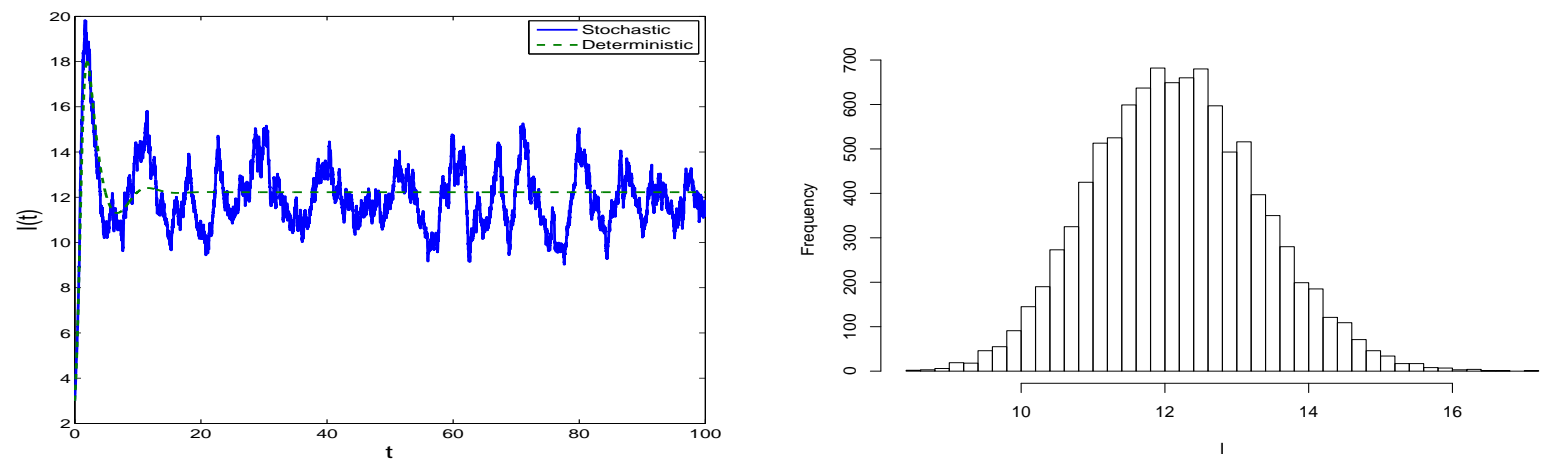

(a) $\sigma_{1}=0.1, \sigma_{2}=0.1$
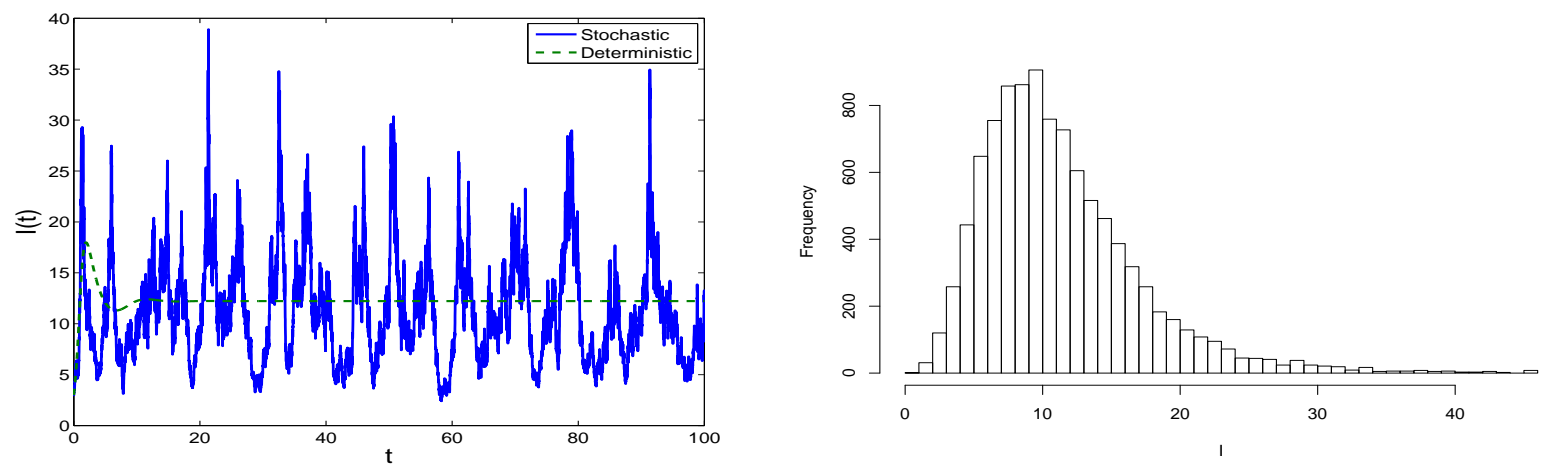

(b) $\sigma_{1}=0.1, \sigma_{2}=0.5$
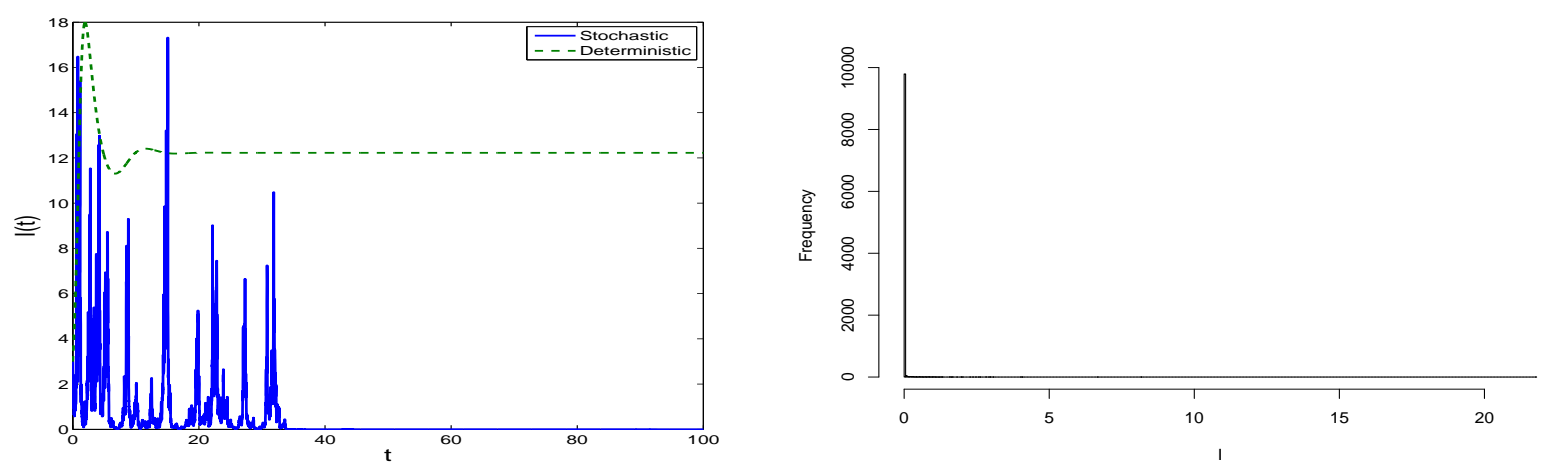

(c) $\sigma_{1}=0.1, \sigma_{2}=2.22$

Figure 2: (LEFT) The evolution of a single path of $I(t)$ for system (3) and its corresponding deterministic model. (RIGHT) Probability densities of the values of the path $I(t)$ for system (3) based on 10000 stochastic simulations. 
after some initial transients the population density fluctuates around the deterministic steady state values $I^{*}=12.2244$. The irregularity of stochastic variation and range of fluctuation increases with the increase in the magnitude of and $\sigma_{2}$. In Fig. 2(c), one can see that large noise $\sigma_{2}=2.3$ induce the extinction of $I(t)$. And in Figure 2(RIGHT), we show the probability densities of the values of the path $I(t)$ at time $t=100$ for system (3) based on 10000 stochastic simulations, and the numerical method for them can be found in [29]. One can find that, in the persistent cases $\sigma_{2}=0.1$ (See Fig. 2(a)) and $\sigma_{2}=0.5$ (See Fig. 2(b)), $I(t)$ distributes around the mean values $I^{*}=12.2244$, and the solution to the SDE model (3) for higher $\sigma_{2}$ (e.g., $\sigma_{2}=0.5$ that the amplitude of fluctuation is remarkable and the distribution of the solution is skewed, while for lower $\sigma_{2}$ (e.g., $\sigma_{2}=0.1$, the amplitude of fluctuation is slight and the oscillations are more symmetrically distributed. More precisely, when $\sigma_{2}=0.1$, the distribution appears closer to a normal distribution (See Fig. 2(a)), but as $\sigma_{2}$ increases to 0.5 , the distribution is positively skewed (See Fig. 2(b)). Obviously, in these two persistent cases, the SDE model (3) has a stationary distribution. However, in the extinct case $\sigma_{2}=2.22$, the mass of the distribution of $I(t)$ is concentrated on the small neighborhood of zero (See Fig. 2(c)). Similar to that in [30], Figure 2 indicates that the small environmental perturbations can generate the irregular cycling phenomena of recurrent diseases, while the large ones will eradicate diseases. This means the small perturbations of the white noise can sustain the irregular recurrence of FIV in cat populations between two pandemics, and larger ones may be beneficial, leading to the extinction of FIV.

\section{Concluding remarks}

In this paper, we have considered the basic features of a simple SI-type epidemic model with varying size in presence of multiplicative noise terms to understand the effects of environmental driving forces on the disease dynamics. The value of this study lies in two aspects:

Mathematically, for the SDE model (3), we have introduced three threshold quantities, the horizontal transmission reproduction number $R_{s}^{h}:=\frac{2 \beta(b-m)+2 k b_{I}-k \sigma_{2}^{2}}{2 k(b+\alpha)}$, and $R_{1}:=b-m-$ 
$\sigma_{1}^{2} / 2, R_{2}:=b_{I}-m-\alpha-\sigma_{2}^{2} / 2$. These three threshold parameters are utilized in identifying the stochastic extinction and persistence (c.f., Theorem 2.2) for the stochastic model (3). In the case of stochastic persistence, we have proved the existence of a stationary distribution (c.f., Theorem 2.3). For the sake of better understanding the stochastic disease dynamics of the SDE model (3), we summarize the theoretical results in the case of $\beta=k$ in the $R_{1} R_{2}$-plane and presented them in Fig. 3.

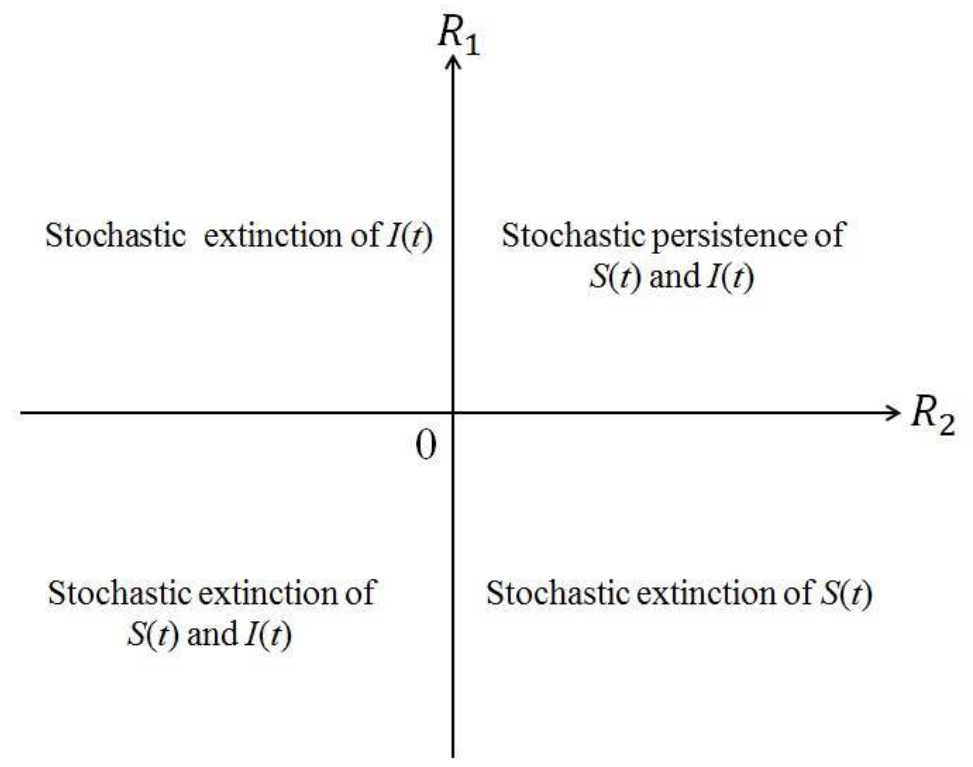

Figure 3: Schematic stochastic dynamics of extinction and endemic of the SDE model (3) in the case of $\beta=k$ in the $\left(R_{1}, R_{2}\right)$-plane.

Epidemiologically, we partially provide the effects of the environment fluctuations on the FIV spreading to the SDE model (3).

1. Large environment fluctuations can suppress the outbreak of disease: For the deterministic model (1), Theorem 2.1 shows that model (1) admits a unique endemic equilibrium $E^{*}$ which is globally asymptotically stable if $T_{0}^{d d}>1$. Notice that $R_{s}^{h}<$ $T_{0}^{d d}$, thus it is possible that $R_{s}^{h}<1<T_{0}^{d d}$. This is the case when the deterministic model (1) is at endemic situation (See, Fig. 1) while the infectious $I(t)$ is extinct in the stochastic model (3) with probability one (see Theorem 2.2 and Fig. 2(c)). This implies that large environment fluctuations can suppress the outbreak of FIV. 
2. The distributions governed by $R_{s}^{h}$ : When the noise intensities $\sigma_{i}(i=1,2)$ are small enough and satisfy the conditions $R_{s}^{h}>1, R_{1}>0$ and $R_{2}>0$, from Theorem 2.3 and Fig. 3, one can see that the environmental noise can drive the solutions of model (3) to oscillate significantly around the endemic steady state (see, Fig. 2), and slight increase of the amplitude of fluctuation leads to the oscillations more symmetrically distributed. In addition, as suggested by Theorems 2.3 and numerical simulation results presented in Fig. 2, the SDE model (3) has an endemic stationary distribution which leads to the stochastic persistence of the FIV disease. And Theorem 2.2 says that under the influence of strong noise intensity, the infectious $I(t)$ will extinct exponentially almost surely; and the susceptible cats $S(t)$ will extinct exponentially almost surely. That is, the extinctions of $I(t)$ or $S(t)$ are induced by the environmental noise, and hence in these cases, we cannot ignore the effect of the noise.

3. The effect of the vertical transmission: In the case of $S(t)$ going to extinct exponentially almost surely, from Theorem 2.2 , one can see that

$$
\liminf _{t \rightarrow \infty} \frac{1}{t} \int_{0}^{t} I d s>\frac{b_{I}-m-\alpha-\frac{\sigma_{2}^{2}}{2}}{k}>0 \text { a.s. }
$$

And from Remark 3.7, we know the distribution of the process $I(t)$ converges to a stationary distribution which has a density $P(I)$ and the mean of density $P(I)$ is

$$
\lim _{\sigma_{2} \rightarrow 0} E(I)=\lim _{\sigma_{2} \rightarrow 0}\left(\frac{b_{I}-m-\alpha}{k}-\frac{\sigma_{2}^{2}}{2 k}\right)=\frac{b_{I}-m-\alpha}{k} .
$$

That is, the susceptible-free situation occurring in this case, which is similar to that in Theorem 2.1 for the deterministic model (1). Also, we can conclude that the susceptible-free situation is induced by the vertical transmission (i.e., $b_{I}>0$ ).

\section{Acknowledgements}

The authors would like to thank the editor and the three referees for their helpful comments. This research was supported by the National Science Foundation of China (61373005). 


\section{References}

[1] N.C. Pedersen, E.W. Ho, M.L. Brown, and J.K. Yamamoto. Isolation of a tlymphotropic virus from domestic cats with an immunodeficiency-like syndrome. Science, 235(4790):790-793, 1987.

[2] J.K. Yamamoto, E.L. Sparger, E.W. Ho, P.R. Andersen, T.P. O'connor, C.P. Mandell, L. Lowenstine, R. Munn, and N.C. Pedersen. Pathogenesis of experimentally induced feline immunodeficiency virus infection in cats. American Journal of Veterinary Research, 49(8):1246-1258, 1988.

[3] T.W. North, G.L. North, and N.C. Pedersen. Feline immunodeficiency virus, a model for reverse transcriptase-targeted chemotherapy for acquired immune deficiency syndrome. Antimicrobial Agents and Chemotherapy, 33(6):915-919, 1989.

[4] R.A. Olmsted, V.M. Hirsch, R.H. Purcell, and P.R. Johnson. Nucleotide sequence analysis of feline immunodeficiency virus: genome organization and relationship to other lentiviruses. Proceedings of the National Academy of Sciences, 86(20):8088-8092, 1989.

[5] M. Bendinelli, M. Pistello, S. Lombardi, A. Poli, C. Garzelli, D. Matteucci, L. Ceccherini-Nelli, G. Malvaldi, and F. Tozzini. Feline immunodeficiency virus: an interesting model for AIDS studies and an important cat pathogen. Clinical Microbiology Reviews, 8(1):87-112, 1995.

[6] J.K. Yamamoto, H. Hansen, E.W. Ho, T.Y. Morishita, T. Okuda, T.R Sawa, R.M. Nakamura, and N.C. Pedersen. Epidemiologic and clinical aspects of feline immunodeficiency virus infection in cats from the continental united states and canada and possible mode of transmission. Journal of the American Veterinary Medical Association, 194(2):213-220, 1989.

[7] F.M. Hilker, M. Langlais, S.V. Petrovskii, and H. Malchow. A diffusive SI model with Allee effect and application to FIV. Mathematical Biosciences, 206(1):61-80, 2007. 
[8] F. Courchamp, D. Pontier, M. Langlais, and M. Artois. Population dynamics of feline immunodeficiency virus within cat populations. Journal of Theoretical Biology, 175(4):553-560, 1995.

[9] L. O'Nell, Mary J. Burkhard, L. Diehl, and E. Hoover. Vertical transmission of feline immunodeficiency virus. AIDS Research and Human Retroviruses, 11(1):171-182, 1995.

[10] W.E. Fitzgibbon, M. Langlais, and J.J. Morgan. A mathematical model of the spread of feline leukemia virus (FeLV) through a highly heterogeneous spatial domain. SIAM Journal on Mathematical Analysis, 33(3):570-588, 2001.

[11] W.E. Fitzgibbon, M. Langlais, and J.J. Morgan. A reaction-diffusion system modeling direct and indirect transmission of diseases. Discrete and Continuous Dynamical Systems-B, 4:893-910, 2004.

[12] W.E. Fitzgibbon, M. Langlais, and J.J. Morgan. A mathematical model for indirectly transmitted diseases. Mathematical Biosciences, 206(2):233-248, 2007.

[13] A. Ducrot, M. Langlais, and P. Magal. Qualitative analysis and travelling wave solutions for the SI model with vertical transmission. Communications in Pure and Applied Analysis, 11:97-113, 2012.

[14] F. Courchamp, L. Berec, and J. Gascoigne. Allee effects in ecology and conservation. Environmental Conservation, 36(1):80-85, 2008.

[15] B. Øksendal. Stochastic Differential Equations: An Introduction with Applications. Springer, 2010.

[16] T.C. Gard. Introduction to stochastic differential equations. Marcel Dekker, New York, 1988.

[17] S. Spencer. Stochastic epidemic models for emerging diseases. PhD thesis, University of Nottingham, 2008. 
[18] L.J.S. Allen. An introduction to stochastic epidemic models. In Mathematical Epidemiology, pages 81-130. Springer, 2008.

[19] J. R. Beddington and R. M. May. Harvesting natural populations in a randomly fluctuating environment. Science, 197(4302):463-465, 1977.

[20] J.E. Truscott and C.A. Gilligan. Response of a deterministic epidemiological system to a stochastically varying environment. Proceedings of the National Academy of Sciences, 100(15):9067-9072, 2003.

[21] X. Mao, G. Marion, and E. Renshaw. Environmental brownian noise suppresses explosions in population dynamics. Stochastic Processes and Their Applications, 97(1):95$110,2002$.

[22] E. Beretta, V. Kolmanovskii, and L. Shaikhet. Stability of epidemic model with time delays influenced by stochastic perturbations. Mathematics and Computers in Simulation, 45(3):269-277, 1998.

[23] X. Mao. Stochastic differential equations and their applications. Horwood, Chichester, 1997.

[24] M. Liu and K. Wang. Survival analysis of stochastic single-species population models in polluted environments. Ecological Modelling, 220(9):1347-1357, 2009.

[25] T. Britton. Stochastic epidemic models: a survey. Mathematical Biosciences, 225(1):24$35,2010$.

[26] A. Gray, D. Greenhalgh, L. Hu, X. Mao, and J. Pan. A stochastic differential equation SIS epidemic model. SIAM Journal on Applied Mathematics, 71(3):876-902, 2011.

[27] A. Lahrouz, L. Omari, and D. Kiouach. Global analysis of a deterministic and stochastic nonlinear SIRS epidemic model. Nonlinear Analysis: Modelling and Control, 16(1):59$76,2011$. 
[28] C. Ji and D. Jiang. Threshold behaviour of a stochastic SIR model. Applied Mathematical Modelling, 38(21):5067-5079, 2014.

[29] Y. Cai, Y. Kang, M. Banerjee, and W. Wang. A stochastic SIRS epidemic model with infectious force under intervention strategies. Journal of Differential Equations, 259(12):7463-7502, 2015.

[30] D. Li, J. Cui, M. Liu, and S. Liu. The evolutionary dynamics of stochastic epidemic model with nonlinear incidence rate. Bulletin of Mathematical Biology, 77(9):1705-1743, 2015.

[31] Y. Zhao, S. Yuan, and J. Ma. Survival and stationary distribution analysis of a stochastic competitive model of three species in a polluted environment. Bulletin of Mathematical Biology, 77:1285-1326, 2015.

[32] P. Van den Driessche and J. Watmough. Reproduction numbers and sub-threshold endemic equilibria for compartmental models of disease transmission. Mathematical Biosciences, 180(1):29-48, 2002.

[33] M. Liu, K. Wang, and Q. Wu. Survival analysis of stochastic competitive models in a polluted environment and stochastic competitive exclusion principle. Bulletin of Mathematical Biology, 73(9):1969-2012, 2011.

[34] P.S. Mandal and M. Banerjee. Stochastic persistence and stationary distribution in a Holling-Tanner type prey-predator model. Physica A: Statistical Mechanics and its Applications, 391(4):1216-1233, 2012.

[35] R. Khasminskii. Stochastic stability of differential equations, volume 66. Springer Science \& Business Media, 2011.

[36] D.J. Higham. An algorithmic introduction to numerical simulation of stochastic differential equations. SIAM Review, 43(3):525-546, 2001. 
[37] D. Pontier. Analyse de la variabilité des traits d'histoire de vie chez les mammiféres. Mémoire d'habilitation à diriger des recherches, 1993. 